\title{
Submicron Features in Higher Manganese Silicide
}

\author{
Yatir Sadia, Mor Elegrably, Oren Ben-Nun, Yossi Marciano, and Yaniv Gelbstein
}

Department of Materials Engineering, Ben-Gurion University of the Negev, Beer Sheva, Israel

Correspondence should be addressed to Yatir Sadia; yatttir@yahoo.com

Received 13 June 2013; Accepted 14 August 2013

Academic Editor: Jung-Kun Lee

Copyright (C) 2013 Yatir Sadia et al. This is an open access article distributed under the Creative Commons Attribution License, which permits unrestricted use, distribution, and reproduction in any medium, provided the original work is properly cited.

\begin{abstract}
The world energy crisis had increased the demand for alternative energy sources and as such is one of the topics at the forefront of research. One way for reducing energy consumption is by thermoelectricity. Thermoelectric effects enable direct conversion of thermal into electrical energy. Higher manganese silicide ( $\left.\mathrm{HMS}, \mathrm{MnSi}_{1.75}\right)$ is one of the promising materials for applications in the field of thermoelectricity. The abundance and low cost of the elements, combined with good thermoelectric properties and high mechanical and chemical stability at high temperatures, make it very attractive for thermoelectric applications. Recent studies have shown that Si-rich HMS has improved thermoelectric properties. The most interesting of which is the unusual reduction in thermal conductivity. In the current research, transmission (TEM) and scanning (SEM) electron microscopy as well as X-ray diffraction methods were applied for investigation of the govern mechanisms resulting in very low thermal conductivity values of an Si-rich HMS composition, following arc melting and hot-pressing procedures. In this paper, it is shown that there is a presence of submicron dislocations walls, stacking faults, and silicon and HMS precipitates inside each other apparent in the matrix, following a high temperature $(0.9 \mathrm{Tm})$ hot pressing for an hour. These are not just responsible for the low thermal conductivity values observed but also indicate the ability to create complicate nano-structures that will last during the production process and possibly during the application.
\end{abstract}

\section{Introduction}

Recent trends in clean energy have given rise to the need for alternative energy sources. One such source is thermoelectric materials, which can utilize waste heat to increase the efficiency of devices with high heat loss. The currently available thermoelectric materials are either not abundant enough for mass production, expensive, toxic, or have too low efficiency to be attractive. The metal silicides are thermoelectric material with low production costs, made from abundant elements, nontoxic and highly stable materials both thermally and mechanically [1]. The most promising $p$-type materials in this family are the higher manganese silicides (HMS) containing several very similar phases including $\mathrm{Mn}_{4} \mathrm{Si}_{7}$, $\mathrm{Mn}_{11} \mathrm{Si}_{19}, \mathrm{Mn}_{15} \mathrm{Si}_{26}$, and $\mathrm{Mn}_{27} \mathrm{Si}_{47}$ [2]. While HMS offers one of the few materials which in addition to good thermoelectric properties, low cost, and high abundance can also operate in atmospheric conditions $[3,4]$, it is yet not high enough in efficiency.

One of the most important measures to the efficiency of a thermoelectric material is the dimensionless figure of merit $(Z T)$ defined as the square of Seebeck coefficient times the temperature over the electric resistivity time of the thermal conductivity. While the all three transport properties are related to the carrier concentration and are therefore optimized mainly by introducing an optimal amount of charge carriers into the materials, research in the field showed [5] that $Z T$ values can be also enhanced by increasing the structure complexity and thereby decreasing the thermal conduction by phonons without adversely affecting the charge carrier concentration.

In recent publications, a low thermal conductivity was realized for HMS samples [6]. In the current research, the influence of apparent submicro features on reduction of the thermal conductivity, following an arc melting and hot pressing, was investigated.

\section{Experimental Procedures}

2.1. Synthesis. The samples were all synthesized from pure silicon $(7 \mathrm{~N})$ and manganese $(99.5 \%)$ pieces. The manganese pieces were cleaned using 50\% hydrochloric acid and 50\% 


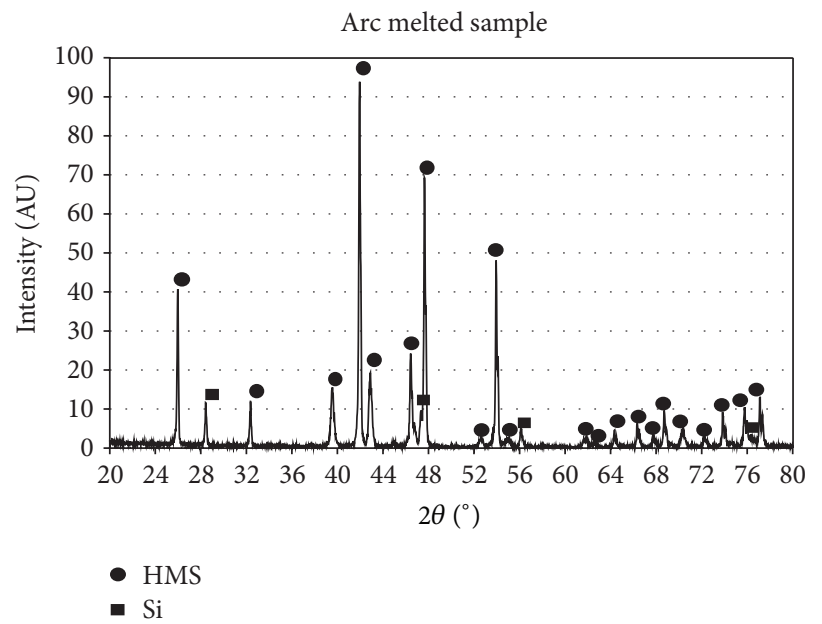

FIGURE 1: X-ray diffraction of eutectic arc melted specimen following hot pressing: $\bullet$ : silicon; $\mathbf{\square}$ : higher manganese silicide peaks.

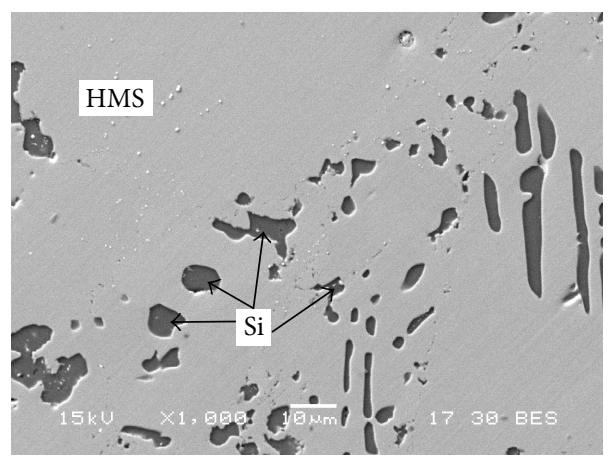

FIGURE 2: SEM micrograph following hot pressing, indicating dark silicon precipitates embedded in a light HMS matrix.

ethanol solution for 10 seconds and flushing in ethanol. The $\mathrm{Mn}$ and Si pieces of the eutectic composition of $\mathrm{MnSi}_{1.91}$ were taken to an arc melting furnace and melted. The resulting ingots were crushed into a powder of $63 \mu \mathrm{m}$ and taken to a hot-press. Hot pressing was done in a graphite die at $1000^{\circ} \mathrm{C}$ for 60 minutes under a mechanical pressure of $50 \mathrm{MPa}$ in an argon atmosphere.

2.2. Structural Measurements. TEM specimens were prepared by cutting a $3 \mathrm{~mm}$ disc using EDM (electric discharge machining) and polished using TEM Minimet Polisher to $100 \mu \mathrm{m}$. The center was polished using a dimple grinder down to a $0.25 \mu \mathrm{m}$ alumina finish. The final preparation was done using a precision ion polishing system. Transmission electron microscopy was performed using a JEOL FasTEM2010 electron microscope.

Scanning electron microscopy was employed for microstructural characterizations using JEOL JSM-5600.

The X-ray data were collected on a Rigaku DMAX 2100 powder diffractometer with a graphite monochromator on the diffracted beam provided by $\mathrm{Cu}$ Ka radiation and operating at $V=40 \mathrm{kV}, I=30 \mathrm{~mA}$.

2.3. Electronic Transport Properties. Seebeck coefficient and the electrical resistivity measurements were carried out in a home-built apparatus up to $\sim 450^{\circ} \mathrm{C}$ (and a heating rate of $3^{\circ} \mathrm{C} / \mathrm{min}$ ) under an Ar atmosphere. For the measurement of the Seebeck coefficient, an auxiliary heater was used to maintain a temperature difference of $10^{\circ} \mathrm{C}$ between the extremities of the samples. The electrical resistivity was measured by the "four-probe" method using an alternating power source of $1 \mathrm{~V} / 50 \mathrm{~Hz}$.

2.4. Thermal Properties. The thermal conductivity was measured from room temperature up to $500^{\circ} \mathrm{C}$ using the flash diffusivity method (LFA 457, Netzsch). Thermal conductivity $(\kappa)$ values were calculated using the equation $\kappa=\alpha \cdot \rho \cdot C_{p}$, where $\alpha$ is the thermal diffusivity, $C_{p}$ is the specific heat (measured using differential scanning calorimetry, STA 449Netzsch), and $\rho$ is the bulk density of the sample.

\section{Results and Discussion}

The XRD pattern shown in Figure 1 indicates the appearance of only the HMS phase with some Si phase remaining, agreeing with the eutectic composition selected. The SEM micrograph in Figure 2 also indicates the existence of only HMS and silicon phases, where $\sim 10-100 \mu \mathrm{m}$ Si precipitates are embedded in the main HMS matrix. 


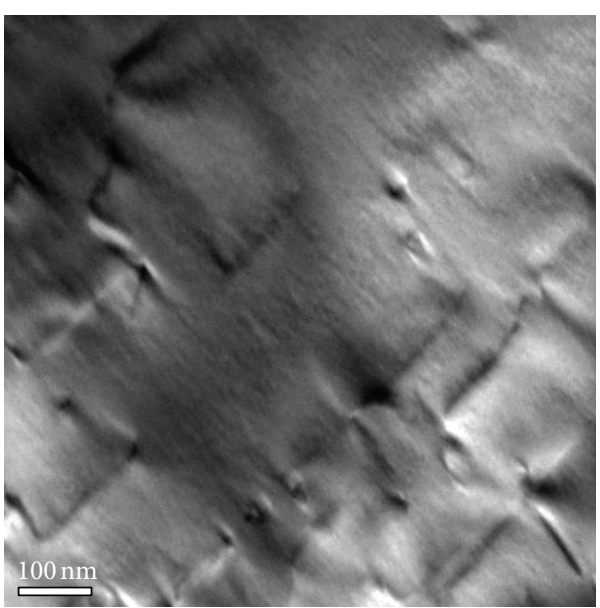

(a)

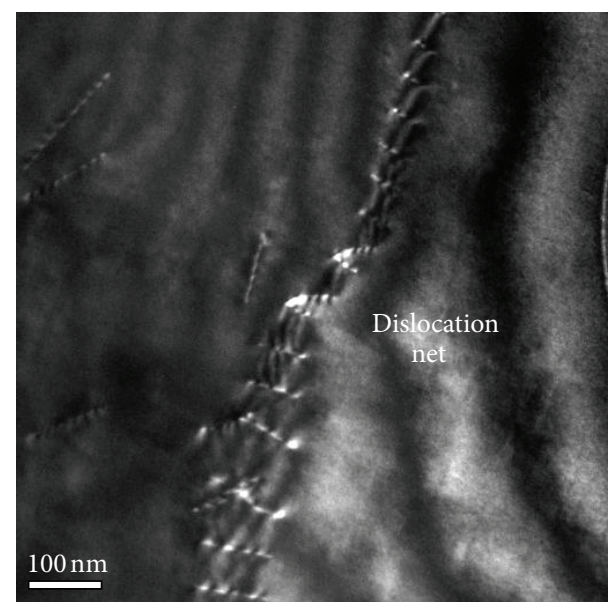

(b)

FIGURE 3: TEM images of the HMS matrix containing (a) a large number of dislocations, (b) dislocation walls.

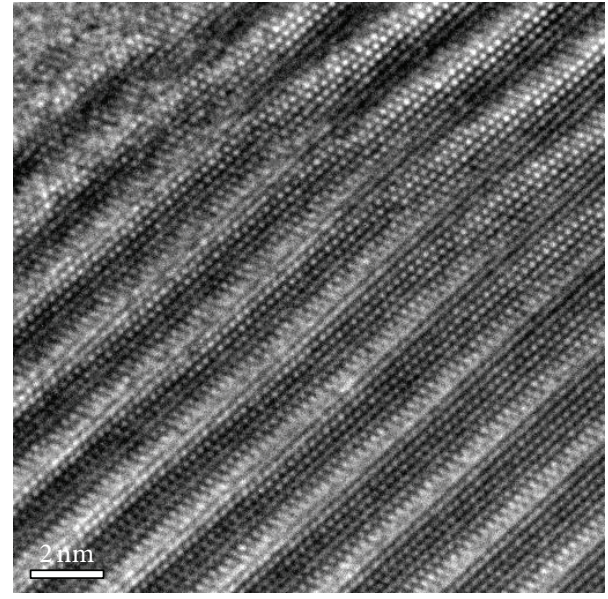

FIGURE 4: TEM lattice image of the hot pressed HMS matrix with a kink showing the fault.

Higher magnification TEM investigation (Figures 3-6) revealed only the appearance of the two types of phases, observed by XRD (Figure 1) and SEM (Figure 2), the white being $\mathrm{Si}$ and the dark being HMS, as were determined by both electrons diffraction and EDS. Yet, several submicron features that can act as phonon scattering centers were also observed.

In Figure 3, a large number of dislocations (Figure 3(a)) and even highly dense dislocation walls (Figure 3(b)), apparent in the HMS matrix, can be seen. Such a high dislocation density is not typical for brittle materials such as HMS, and their presence can serve as an efficient phonon scattering source. One more predominant feature can be seen in the lattice image in Figure 4. This type of deviation from the straight lattice was reported in [2] by Ye and Amelinckx. This feature is an extra silicon plane in the middle of the HMS lattice, a special type of stacking fault or dislocation which predominates in HMS.
When looking for larger defects, two main interesting phenomena were observed: submicron precipitates of $\mathrm{Si}$ in between the HMS grains and submicron precipitates of HMS inside silicon grains. Figure 5 illustrates two typical Si submicron precipitates between two adjacent HMS grains, one in a triple junction (Figure 5(a)) and one between two grains (Figure 5(b)). The presence of submicron precipitates following the high temperature hot-pressing procedure, at $0.9 \mathrm{Tm}$ of the HMS phase, can provide evidence to the fact that, due to the low diffusion rates of the HMS phase, the submicron features can be stable at or above the working temperature $\left(660^{\circ} \mathrm{C}\right.$ in air $[3,4]$ and up to about $750^{\circ} \mathrm{C}$ in argon). This evidence is also coupled with the HMS precipitates in an Si grain that can be seen in Figure 6. In this figure, a trail of precipitates is observed (Figure 6(a)), which seems to be parallel to the grain boundary shown in Figure 6(b). This can be explained by very fine HMS powder, getting trapped between two silicon grains during the hotpressing procedure. While the silicon has high diffusion rates in the matrix enhancing the grain boundary movement, the HMS apparently stays where the original grain boundary existed. This further supports the fact that the observed submicron features can be stable at high temperatures.

The described above submicron features in the investigated HMS sample seem to arise from the powdering process, while keeping their size after a long hot-press process. The advantage that can be gained by reducing the lattice thermal conductivity is highly dependent on the ability to retain submicron features during long operations. While very few materials can keep their original submicron features upon high temperature exposure, due to thermodynamic forces, it seems that HMS is the perfect candidate for retaining of such features.

The measured Seebeck coefficient and electrical conductivity values are shown in Figure 7. The positive Seebeck coefficients clearly indicate that the investigated composition is a $p$-type semiconductor, while the increased/decreased trend of Seebeck coefficient/electrical conductivity with increasing 


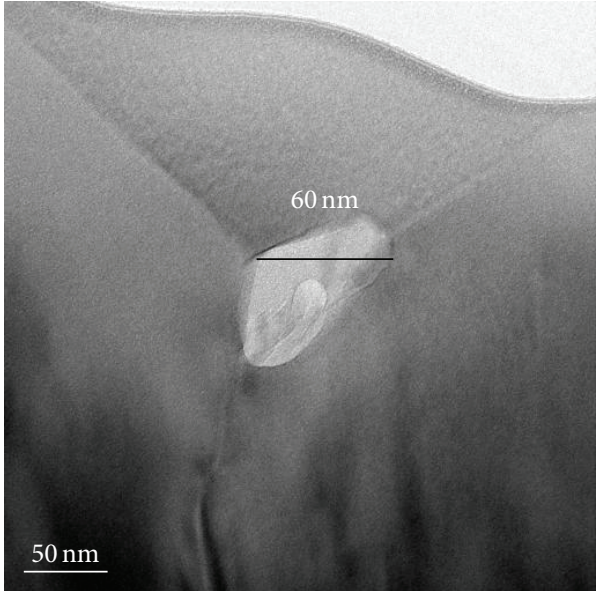

(a)

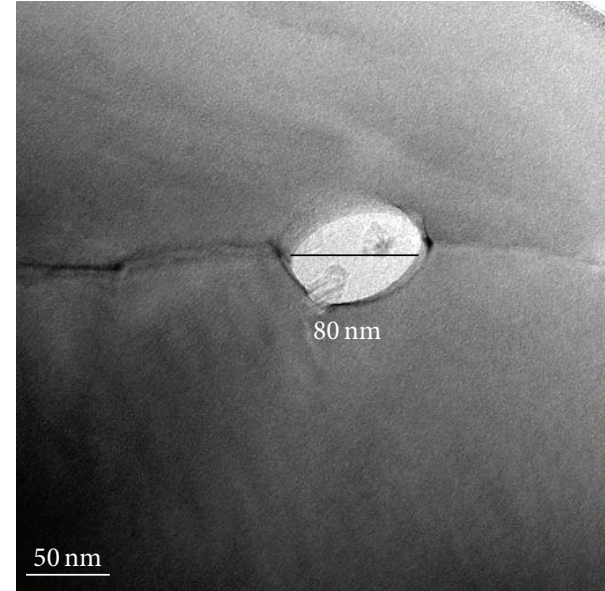

(b)

FIGURE 5: TEM images of the HMS matrix containing (a) a $60 \mathrm{~nm}$ silicon precipitate in a triple junction, (b) an $80 \mathrm{~nm}$ precipitate of silicon between two HMS grains.

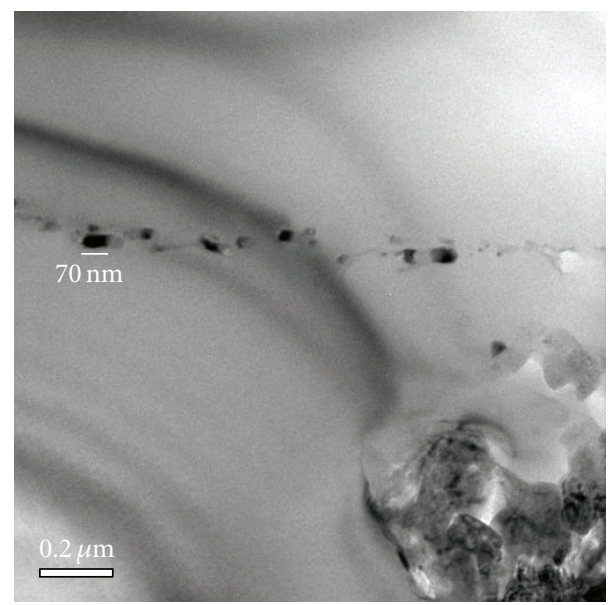

(a)

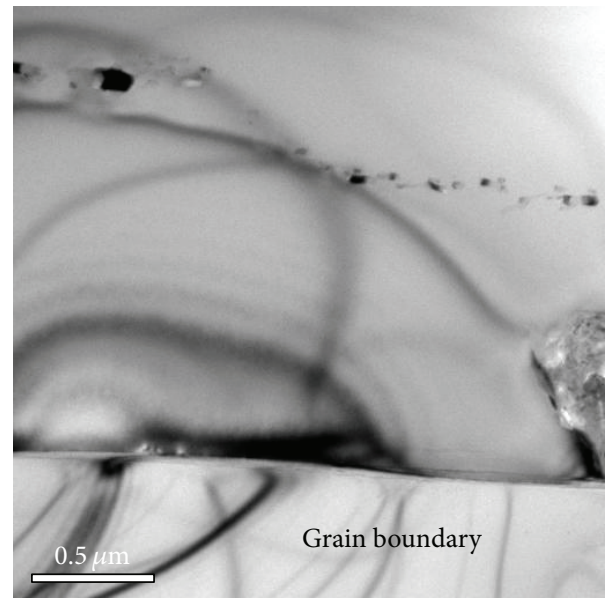

(b)

FIGURE 6: TEM images of the HMS matrix containing (a) a trail of submicron grains within the silicon matrix, (b) a parallel grain boundary.

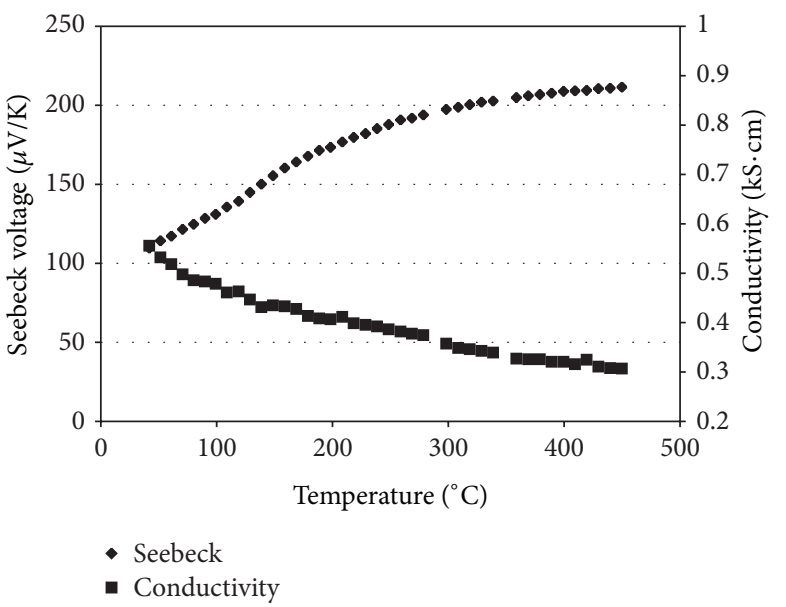

FIGURE 7: The Seebeck coefficient and the electrical conductivity values following arc melting and hot pressing.

of the temperature clearly indicates a metallic nature, highlighting the highly degenerate nature of the semiconductor. Figure 8 shows the total and lattice thermal conductivities as well as the dimensionless thermoelectric figure of merit, $Z T$, values obtained following arc melting and hot pressing. The electronic contribution to the thermal conductivity, $\kappa_{e}$, was calculated using the Wiedemann-Franz relation (1) and the measured electrical conductivity values (Figure 7). The figure shows that the phonon contribution to the thermal conductivity outweighs the electronic contribution, meaning that, even if some electron scattering occurs, it should not be highly significant:

$$
\kappa_{e}=L \sigma T,
$$




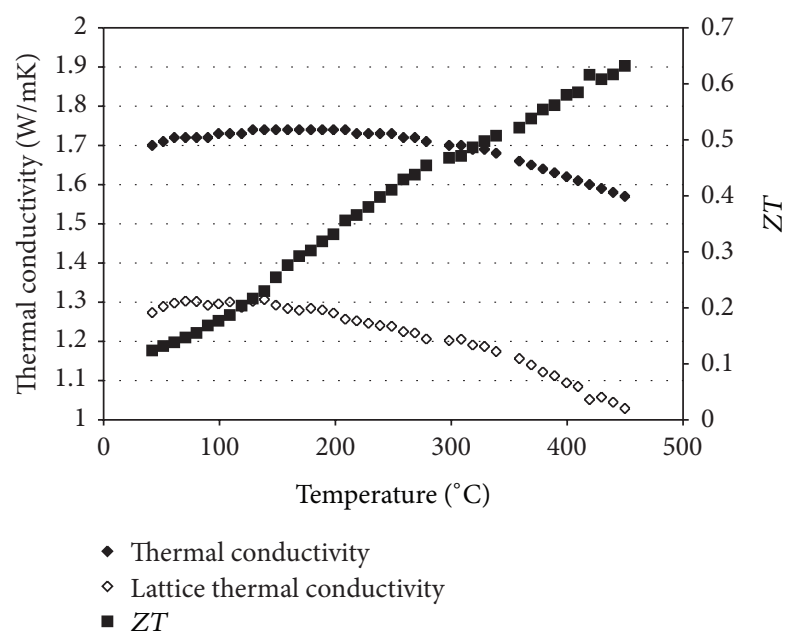

FIgURE 8: Total and lattice thermal conductivities as well as the dimensionless figure of merit, $Z T$, following arc melting and hot pressing.

where $L$ and $T$ are Lorenz numbers which in highly degenerated semiconductors are constants equal to $2.44 \cdot 10^{-8}$ $\mathrm{W} \Omega \mathrm{K}^{-2}$ and the absolute temperature, respectively. The lattice thermal conductivity shown in Figure 8 was calculated by subtracting the electronic component of the thermal conductivity from the total thermal conductivities.

It can be seen that very low lattice thermal conductivity values of $\sim 1-1.3 \mathrm{~W} / \mathrm{mK}$ were obtained for the investigated temperature range, which can be attributed to the submicron features observed and retained following the high temperature hot-pressing procedure (Figures 3-6), resulting in very high $Z T$ values with a maximal value of $\sim 0.63$, observed at $450^{\circ} \mathrm{C}$.

\section{Conclusions}

Higher manganese silicide-based samples were prepared by arc melting, hand milling, and hot pressing. Of the samples TEM, SEM, and XRD specimens were prepared and examined. The specimen showed various submicron features which can contribute to the low thermal conductivity measured. These features include dislocation walls, stacking faults, and nanoclusters of both HMS inside the Si matrix and Si inside the HMS matrix. These features were retained following the hot-pressing procedure, which was performed at 0.9 of the homologues temperature of HMS, clearly indicating promise for durability of the features during long operations.

\section{Acknowledgments}

The authors would like to acknowledge Engineer A. Jarashnely for his help and expertise with XRD characterization and Dr. S. Remenik for the help and expertise with TEM characterization.

\section{References}

[1] C. B. Vining, CRC Handbook of Thermoelectrics, CRC Press, Boca Raton, Fla, USA, 1995.

[2] H. Q. Ye and S. Amelinckx, "High-resolution electron microscopic study of manganese silicides MnSi2-x," Journal of Solid State Chemistry, vol. 61, no. 1, pp. 8-39, 1986.

[3] S. Okada, T. Shishido, M. Ogawa et al., "MnSi and MnSi2x single crystals growth by Ga flux method and properties," Journal of Crystal Growth, vol. 229, no. 1, pp. 532-536, 2001.

[4] S. Okada, T. Shishido, Y. Ishizawa et al., "Crystal growth by molten metal flux method and properties of manganese silicides," Journal of Alloys and Compounds, vol. 317-318, pp. 315319, 2001.

[5] G. J. Snyder and E. S. Toberer, "Complex thermoelectric materials," Nature Materials, vol. 7, no. 2, pp. 105-114, 2008.

[6] Y. Sadia and Y. Gelbstein, "Silicon-rich higher manganese silicides for thermoelectric applications," Journal of Electronic Materials, vol. 41, no. 6, pp. 1504-1508, 2012. 

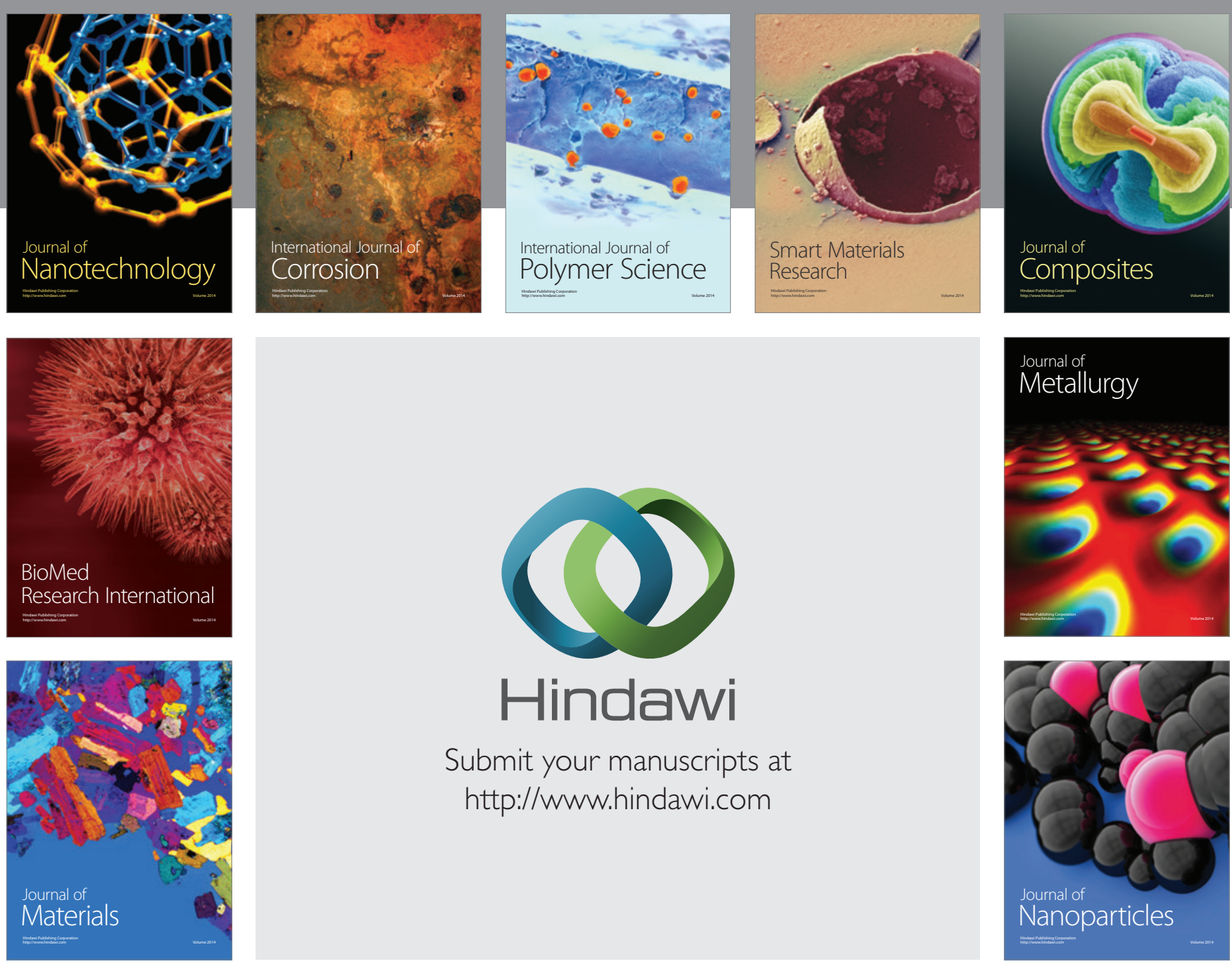

Submit your manuscripts at http://www.hindawi.com
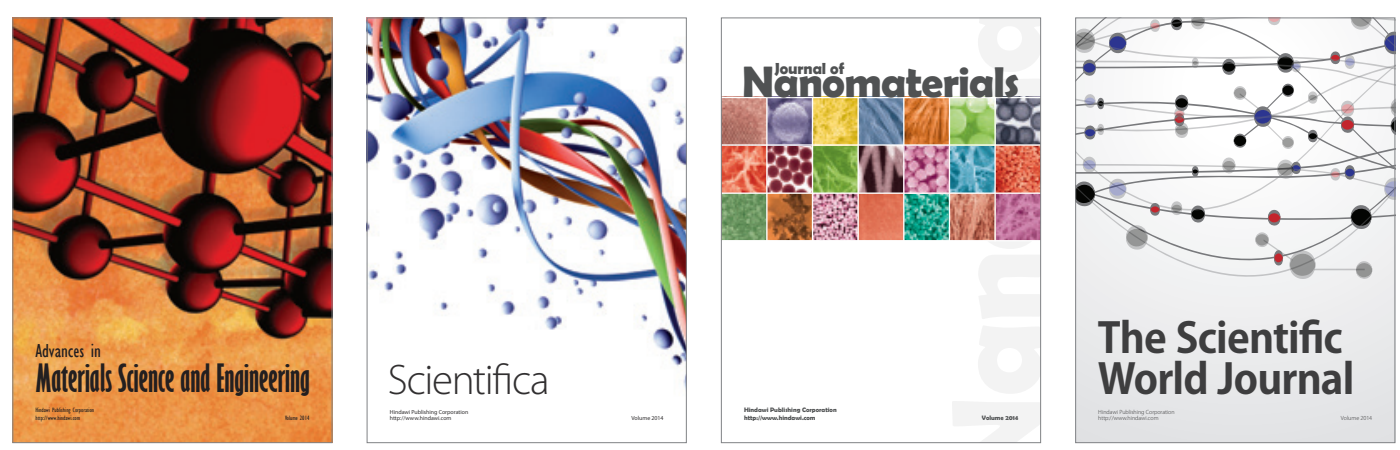

\section{The Scientific World Journal}
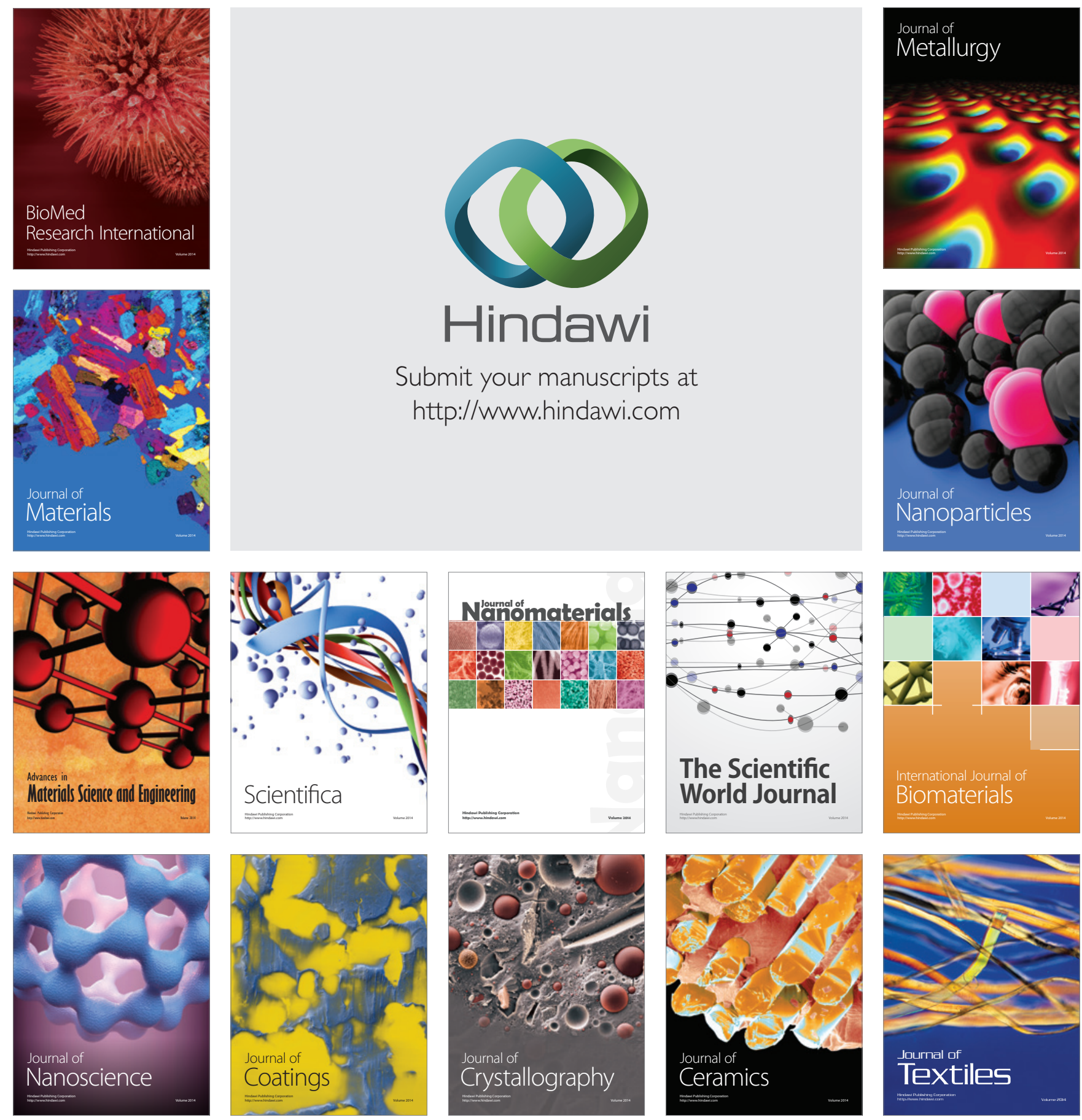